\title{
Some Algebraic Properties of the Wilson Loop
}

\author{
Han Li $\mathbb{D}^{1},{ }^{1}$ M. Nadeem, ${ }^{2}$ M. A. Ali, ${ }^{3}$ and H. Mutee ur Rehman $\mathbb{D}^{4}$ \\ ${ }^{1}$ School of Mathematics, Chengdu Normal University, Chengdu 611130, China \\ ${ }^{2}$ Lahore Garrison University, Lahore 54000, Pakistan \\ ${ }^{3}$ Virtual University of Pakistan, Lahore 54000, Pakistan \\ ${ }^{4}$ Department of Mathematics, Division of Science and Technology, University of Education, Lahore 54000, Pakistan
}

Correspondence should be addressed to Han Li; woshilihanlaoshi@sina.com

Received 10 September 2021; Accepted 1 December 2021; Published 4 January 2022

Academic Editor: Yuan Yi

Copyright $\odot 2022 \mathrm{Han} \mathrm{Li} \mathrm{et} \mathrm{al.} \mathrm{This} \mathrm{is} \mathrm{an} \mathrm{open} \mathrm{access} \mathrm{article} \mathrm{distributed} \mathrm{under} \mathrm{the} \mathrm{Creative} \mathrm{Commons} \mathrm{Attribution} \mathrm{License,} \mathrm{which}$ permits unrestricted use, distribution, and reproduction in any medium, provided the original work is properly cited.

In this article, some algebraic properties of the Wilson loop have been investigated in a broad manner. These properties include identities, autotopisms, and implications. We use some equivalent conditions to study the behavior of holomorphism of this loop. Under the shadow of this holomorphism, we are able to observe coincident loops.

\section{Introduction and Definitions}

A nonempty set is defined to be a groupoid relative to the operation (.) if and only if to each ordered pair $(\alpha, \beta)$ of elements of $L$, there exists a uniquely defined element $\alpha$. $\beta \in L$ (or simply $\alpha \beta \in L$ ). If $\mu \in L$ is a fixed element, then there exist two single-valued mappings $L_{\mu}, R_{\mu}$ of $L$ into itself by

$$
(\alpha) L_{\mu}=\mu \alpha \text { and }(\alpha) R_{\mu}=\alpha \mu \text { for all } \alpha \in L .
$$

An element $1 \in L$ is said to be unit of $L$ if for each $\alpha \in L$, we have the identity law $\alpha 1=1 \alpha=\alpha$. A unit if exists is unique. An element $\mu \in L$ is said to be left nonsingular if $L_{\mu}$ is a permutation of $L$ and similarly an element $\mu$ of $L$ is said to be right nonsingular if $R_{\mu}$ is a permutation of $L$. A system $(L, \cdot, \backslash, /)$ is called a quasigroup provided each of $(L, \cdot),(L, \backslash)$, and $(L, /)$ is a groupoid and

$$
\begin{aligned}
& \alpha\left(\frac{\alpha}{\beta}\right)=\beta,\left(\frac{\alpha}{\alpha \beta}\right)=\beta, \\
& \left(\frac{\alpha \beta}{\beta}\right)=\alpha,\left(\frac{\alpha}{\beta}\right) \beta=\alpha .
\end{aligned}
$$

For all, $\alpha, \beta \in L$. For a quasigroup $L$, we can associate a permutation $T_{\alpha}: L \longrightarrow L$ defined by $(\beta) T_{\alpha}=\alpha \beta / \alpha, \forall \alpha$, $\beta \in L$. A quasigroup which admits identity law is called a loop. For each $\alpha \in L$, the elements $\alpha^{\rho}=(\alpha) J_{\rho}, \alpha^{\lambda}=(\alpha) J_{\lambda}$ such that $\alpha \alpha^{\rho}=e^{\rho}, \alpha^{\lambda} \alpha=e^{\lambda}$ are called the right and left inverses of $\alpha$ under right $J_{\rho}: L \longrightarrow L$ and left $J_{\lambda}: L \longrightarrow L$ inverse permutations on $L$, respectively. A loop satisfying Wilson identity in [1] is called Wilson loop. From Table 1 of Wilson loop, we have $11 \cdot 10=0$ which shows 10 is the right inverse of 11 with right multiplicative identity 0 . For a loop $L$, the right and left inverses of an element of $L$ are different in general but right $e^{\rho}$ and left $e^{\lambda}$ identities are always same. It is not easy to control this identity algebraically due to the fact that there are three variables appearing at the right and two at the left side. A loop $L$ with weak associativity $(\alpha \beta)(\gamma \alpha)=(\alpha(\beta \gamma)) \alpha$ is called Moufang loop for all $\alpha, \beta, \gamma \in L$. Nucleus of the loop $L$ is the intersection of the left $\aleph_{\lambda}=\{\alpha \in L: \alpha(\beta \gamma)=(\alpha \beta) \gamma, \forall \beta, \gamma \in L\}, \quad$ middle $\aleph_{\chi}=$ $\{\beta \in L: \alpha(\beta \gamma)=(\alpha \beta) \gamma, \forall \alpha, \gamma \in L\}$, and right $\aleph_{\rho}=\{\gamma \in L$ : $\alpha(\beta \gamma)=(\alpha \beta) \gamma, \forall \alpha, \beta \in L\}$ nuclei of $L$ denoted by $\aleph(L)$. An ordered triple $(\Phi, \Psi, \xi)$ of permutations of loop $L$ is in the group of autotopisms, AUT $(L)$, of $L$ if and only if $(\alpha) \Phi(\beta) \Psi=(\alpha \beta) \xi, \forall \alpha, \beta \in L$. If these bijections are coincident, then $\Phi$ is automorphism in group $\operatorname{AUM}(L)$. To every loop $L$ with $\operatorname{AUM}(L)$, there corresponds another set $H(L)$. If we define $\diamond$ on $H(L)$ such that $(\Phi, \alpha) \diamond(\Psi, \beta)=$ $(\Phi \Psi, \alpha \Psi \beta)$ for all $(\Phi, \alpha),(\Psi, \beta) \in H(L),\{\Phi, \Psi\} \subseteq \operatorname{AUM}(L)$, 
and $\alpha, \beta \in L$, then $H(L)$ is a loop and called the holomorph of $L$.

Inspired by the work on Moufang loop in [1], Goodaire and Robinson [2] introduced a new understanding of Wilson loop with the help of weak inverse property loop and conjugacy closed loop. It is shown in [1] that a Moufang loop is Wilson loop if and only if $\alpha^{2} \in \aleph(L)$ for all $\alpha \in L$. In [3], the concept of holomorph of a loop is given by Bruck. An extension work on holomorphism of a loop is done in [4].

In the present paper, it is shown that we can obtain loop isotopes of Wilson loop under different autotopisms. In this way, we are able to give new forms of Wilson loops by opposite loops, and finally, we see conditions to prove that holomorph of a Wilson loop is a Wilson loop.

We finish this section by giving an example of a noncommutative and nonassociative Wilson loop of order 12 in the form of Table 1.

\section{Results and Identities}

A permutation $P$ of a loop $L$ is known as pseudoautotopism of $L$ if and only if there exists an element $c \in L$ such that $\left(P, P R_{c}, P R_{c}\right)$ is an autotopism of $L$. The element $c$ is called a companion of $P$. The pseudoautotopisms form a group denoted by $\operatorname{PS}(L)$. The following proposition portrays that the pseudoautotopism is helpful to find a loop which is an isotope of the Wilson loop.

Proposition 1. Let $L$ be a Moufang loop with the property $\alpha^{2} \in \aleph(L)$ for all $\alpha \in L$ and $\theta \in P S_{\lambda}(L)$ with companion $c$. Then, we have $\left(\theta L_{c^{3}}, \theta, \theta L_{c^{3}}\right)$ as an autotopism of the Wilson loop $L$.

Proof. Because $\theta \in \mathrm{PS}_{\lambda}(L)$ with companion $c$, so

$$
(c \cdot \alpha \theta)(\beta \theta)=c \cdot(\alpha \beta) \theta \text { for all } \alpha, \beta \in L \text {. }
$$

Consider $\quad(n c \cdot \alpha \theta)(\beta \theta)=(n \cdot c \alpha \theta)(\beta \theta)=n((c \cdot \alpha \theta)$ $(\beta \theta))=n(c(\alpha \beta) \theta)=(n c)(\alpha \beta) \theta$ which shows that $n c$ is also a companion of $\theta$. By [1], $L$ is the Wilson loop, so $\aleph_{\lambda}=\aleph_{\chi}=\aleph_{\rho}$. With the given property, $\alpha^{2} \in \aleph(L)$ for all $\alpha \in L$. So, $c^{2} \cdot c$ is a companion of $\theta$, and in Moufang loop $L$, $\alpha^{3}$ for all $\alpha \in L$ is well-defined. Therefore, $\left(c^{3} \cdot \alpha \theta\right)$ $(\beta \theta)=c^{3} \cdot(\alpha \beta) \theta \Rightarrow\left(\theta L_{c^{3}}, \theta, \theta L_{c^{3}}\right)$ is an autotopism of the Wilson loop $L$.

We are able to find a class of loop isotopes of the Wilson loop with respect to different natural numbers. A loop $L$ has the isotopy-isomorphy property provided each loop isotopic to $L$ is isomorphic to $L$. Such a loop is called G-loop, and in [1], it is proved that the Wilson loop is an example of the G-loop.

Lemma 1. If $L$ is a Wilson loop and $\theta$ is the right pseudoautotopism on $L$ with companion $c$, then $c$ is in the nucleus of $L$ as well as in the nucleus of the loop isotope of L. Moreover, $\theta$ has companions $c^{i}$ (not all distinct) for natural number $i$.

Proof. Let $L$ be isomorphic to a loop isotope $L(1, c, \diamond)$ under the isomorphism $\pi:(L,.) \longrightarrow L(1, c, \diamond)$ defined by $(\alpha) \pi=$ $\alpha c$ for all $\alpha \in L$ where $c$ is a companion of $\theta$. By [1], we have $c \in \aleph_{\rho}$. But, in Wilson loop, three nuclei are coincident, so $c \in \aleph(L)$. Because $c$ is an identity of $L(1, c, \diamond)$ and $\aleph$ $(L((1, c, \diamond))$ is a subgroup of $L(1, c, \diamond), \Rightarrow c \in \aleph(L(1, c, \diamond))$. Since $\theta$ has companions $c$ as well as $n c$ for all $n \in \aleph(L)$, therefore $c \cdot c, c^{2} \cdot c, \ldots$ are the companions (not all distinct) of $\theta$. Thus, $\theta$ has companions $c^{i}$ for natural number $i$.

Note that the powers of companions play an important role as well as the left and the right inverse permutations are also important under an arbitrary autotopism.

Lemma 2. If $(U, V, W)$ is an autotopism of the Wilson loop $L$, then $\left(T_{\alpha} V, L_{\alpha} J_{\lambda} W J_{\rho}, \quad L_{\alpha} J_{\lambda} U J_{\rho}\right)$ and $\left(R_{\alpha} V, T_{\alpha}^{-1} J_{\lambda}\right.$ $W J_{\rho}, R_{\alpha} J_{\lambda} U J_{\rho}$ ) are also autotopisms on $L$ for all $\alpha, \beta, \gamma \in L$.

Proof. Let $\left(T_{\alpha}, L_{\alpha}, L_{\alpha}\right)$ and $\left(R_{\alpha}, T_{\alpha}^{-1}, R_{\alpha}\right)$ be autotopisms on $L$, so $(\beta) T_{\alpha}(\gamma) L_{\alpha}=(\beta \gamma) L_{\alpha}, \Rightarrow(\alpha \beta / \alpha)(\alpha \gamma)=\alpha(\beta \gamma), \Rightarrow(\gamma) L_{\alpha}$ $L_{\alpha \beta / \alpha}=(\gamma) L_{\beta} L_{\alpha}, \Rightarrow L_{\alpha \beta / \alpha}=L_{\alpha}^{-1} L_{\beta} L_{\alpha}$.

Also, $\quad(\beta) R_{\alpha}(\gamma) T_{\alpha}^{-1}=(\beta \gamma) R_{\alpha}, \quad \Rightarrow(\gamma \alpha)(\alpha / \beta \alpha)=(\gamma \beta) \alpha$, $\Rightarrow(\gamma) R_{\alpha} R_{\alpha /(\beta \alpha)}=(\gamma) R_{\beta} R_{\alpha}, \Rightarrow R_{\alpha /(\beta \alpha)}=R_{\alpha}^{-1} R_{\beta} R_{\alpha}$. In [5], $\left(V, J_{\lambda} W J_{\rho}, J_{\lambda} U J_{\rho}\right)$ is also autotopism of $L$. Therefore, we obtain $\left(T_{\alpha}, L_{\alpha}, L_{\alpha}\right)\left(V, J_{\lambda} W J_{\rho}, J_{\lambda} U J_{\rho}\right)=\left(T_{\alpha} V, L_{\alpha} J_{\lambda} W J_{\rho}\right.$, $\left.L_{\alpha} J_{\lambda} U J_{\rho}\right)$. Hence, we have $\left(R_{\alpha}, T_{\alpha}^{-1}, R_{\alpha}\right) \quad\left(V, J_{\lambda} W J_{\rho}\right.$, $\left.J_{\lambda} U J_{\rho}\right)=\left(R_{\alpha} V, T_{\alpha}^{-1} J_{\lambda} W J_{\rho}, R_{\alpha} J_{\lambda} U J_{\rho}\right)$.

We have autotopisms in the form of the left and right translations for the special subclass and Wilson loops of exponents 2 without depending upon the given arbitrary autotopism.

Proposition 2. If L is the Wilson loop of exponent 2, then the following are autotopisms on $\mathrm{L}$ :
(1) $\left(R_{\alpha}, R_{(\alpha)}, R_{\alpha}\right)$
(2) $\left(L_{(\alpha)}, L_{\alpha}, L_{\alpha}\right)$
(3) $\left(R_{\alpha} L_{(\alpha)}, R_{(\alpha)} L_{\alpha}, R_{\alpha} L_{\alpha}\right)$
(4) $\left(L_{(\alpha)} R_{\alpha}, L_{\alpha} R_{(\alpha)}, L_{\alpha} R_{\alpha}\right)$

Proof. Wilson identity in the form of left inverse is $(\beta \alpha)^{\lambda} \alpha=$ $((\gamma \beta) \alpha)^{\lambda}(\gamma \alpha)$ and serves $\beta(\gamma \beta)^{\rho}=\gamma^{\rho}$ for every $\alpha, \beta, \gamma \in L$. Replacing $\gamma$ by $(\gamma \beta \cdot \alpha)^{\lambda}$ and $\beta$ by $\gamma \alpha$ in the later identity $(\gamma \alpha)\left((\gamma \beta \cdot \alpha)^{\lambda}(\gamma \alpha)\right)^{\rho}=\left((\gamma \beta \cdot \alpha)^{\lambda}\right)^{\rho}, \quad \Rightarrow(\gamma \alpha)\left((\beta \cdot \alpha)^{\lambda} \alpha\right)^{\rho}$ $=(\gamma \beta \cdot \alpha)$. It implies that $\left(R_{\alpha}, R_{\alpha} J_{\lambda} R_{\alpha} J_{\rho}, R_{\alpha}\right)$ is an autotopism on Wilson loop $L$. By using the same behavior, we can show that $\left(L_{\alpha} J_{\rho} L_{\alpha} J_{\lambda}, L_{\alpha}, L_{\alpha}\right)$ is another autotopism for this $L$. Due to the exponent $2, J_{\lambda}$ and $J_{\rho}$ become identity mapping. Therefore, $\left(R_{\alpha}, R_{\alpha} R_{\alpha}, R_{\alpha}\right)$ and $\left(L_{\alpha} L_{\alpha}, L_{\alpha}, L_{\alpha}\right)$ are the autotopisms of $L$. So, $\left(R_{\alpha}, R_{(\alpha)}, R_{\alpha}\right)$ and $\left(L_{(\alpha)}, L_{\alpha}, L_{\alpha}\right)$ are the members of $\operatorname{AUT}(L)$. Equations (3) and (4) are trivial by definition of AUT $(L)$.

Theorem 1. Let $L$ be a Wilson loop and $\alpha, \beta, \gamma \in L$. The following identities hold:
(1) $L_{\beta} J_{\lambda} R_{\beta} L_{\alpha} L_{\beta} L_{\alpha}^{-1}=J_{\lambda} L_{\alpha / \beta \alpha}$
(2) $L_{\beta} J_{\lambda} R_{\beta} R_{\alpha} R_{\beta} R_{\alpha}^{-1}=J_{\lambda} R_{\alpha \beta / \alpha}$
(3) $L_{\beta} J_{\lambda} R_{\beta} R_{\beta} L_{\alpha} R_{\alpha \beta}^{-1}=J_{\lambda} T_{\alpha}$ 
TABLe 1: An example of a noncommutative and nonassociative Wilson loop of order 12.

\begin{tabular}{|c|c|c|c|c|c|c|c|c|c|c|c|c|}
\hline$c$ & 0 & 1 & 2 & 3 & 4 & 5 & 6 & 7 & 8 & 9 & 10 & 11 \\
\hline 0 & 0 & 1 & 2 & 3 & 4 & 5 & 6 & 7 & 8 & 9 & 10 & 11 \\
\hline 1 & 1 & 2 & 0 & 4 & 5 & 3 & 7 & 8 & 6 & 10 & 11 & 9 \\
\hline 2 & 2 & 0 & 1 & 5 & 3 & 4 & 8 & 6 & 7 & 11 & 9 & 10 \\
\hline 3 & 3 & 4 & 5 & 0 & 1 & 2 & 10 & 11 & 9 & 8 & 6 & 7 \\
\hline 4 & 4 & 5 & 3 & 1 & 2 & 0 & 11 & 9 & 10 & 6 & 7 & 8 \\
\hline 5 & 5 & 3 & 4 & 2 & 0 & 1 & 9 & 10 & 11 & 7 & 8 & 6 \\
\hline 6 & 6 & 7 & 8 & 11 & 9 & 10 & 0 & 1 & 2 & 4 & 5 & 3 \\
\hline 7 & 7 & 8 & 6 & 9 & 10 & 11 & 1 & 2 & 0 & 5 & 3 & 4 \\
\hline 8 & 8 & 6 & 7 & 10 & 11 & 9 & 2 & 0 & 1 & 3 & 4 & 5 \\
\hline 9 & 9 & 10 & 11 & 7 & 8 & 6 & 5 & 3 & 4 & 0 & 1 & 2 \\
\hline 10 & 10 & 11 & 9 & 8 & 6 & 7 & 3 & 4 & 5 & 1 & 2 & 0 \\
\hline 11 & 11 & 9 & 10 & 6 & 7 & 8 & 4 & 5 & 3 & 2 & 0 & 1 \\
\hline
\end{tabular}

(4) $L_{\beta} J_{\lambda} R_{\beta} L_{\beta} R_{\alpha} L_{\beta \alpha}^{-1}=J_{\lambda} T_{\alpha}^{-1}$

(5) $R_{\beta} J_{\lambda} L_{\beta} L_{\alpha}^{-1} R_{\gamma}^{-1} R_{\beta} L_{\alpha}=J_{\lambda} R_{\alpha \gamma}^{-1} R_{\alpha \beta}$

(6) $R_{\beta} J_{\lambda} L_{\beta} R_{\alpha}^{-1} L_{\gamma}^{-1} L_{\beta} R_{\alpha}=J_{\lambda} L_{\gamma \alpha}^{-1} L_{\beta \alpha}$

Proof. (1) In [2], it is shown that $L$ is conjugacy closed loop (CCL) and weak inverse property loop (WIPL). We have $(\alpha)$ $L_{\beta} J_{\lambda} R_{\beta}=(\beta \alpha) J_{\lambda} R_{\beta}=(\beta \alpha)^{\lambda} R_{\beta}=(\beta \alpha)^{\lambda} \beta=\alpha^{\lambda}=(\alpha) J_{\lambda}$ and $L_{\beta} J_{\lambda} R_{\beta}=J_{\lambda}$. Thus, in case of Wilson loop, we have $L_{\beta} J_{\lambda} R_{\beta} L_{\alpha} L_{\beta} L_{\alpha}^{-1}=J_{\lambda} L_{\alpha / \beta \alpha}$.

For (3), since by CCL, we have $L_{\alpha}^{-1} L_{\gamma} L_{\alpha}=L_{\alpha \gamma / \alpha}, \Rightarrow(\beta)$ $L_{\gamma} \quad L_{\alpha}=(\beta) \quad L_{\alpha} \quad L_{\alpha \gamma / \alpha}, \Rightarrow \alpha(\gamma \beta)=(\alpha \gamma / \alpha)(\alpha \beta), \Rightarrow(\gamma) R_{\beta}$ $L_{\alpha}=(\gamma) T_{\alpha} R_{\alpha \beta}, \Rightarrow R_{\beta} L_{\alpha} R_{\alpha \beta}^{-1}=T_{\alpha}$. By WIPL, $L_{\beta} J_{\lambda} R_{\beta}=J_{\lambda}$. Thus, $L_{\beta} J_{\lambda} R_{\beta} R_{\beta} L_{\alpha} R_{\alpha \beta}^{-1}=J_{\lambda} T_{\alpha}$.

For (5), $T_{\alpha}=R_{\beta} L_{\alpha} R_{\alpha \beta}^{-1}, \Rightarrow T_{\alpha}=R_{\gamma} L_{\alpha} R_{\alpha \gamma}^{-1}, \Rightarrow R_{\beta} L_{\alpha}$ $R_{\alpha \beta}^{-1}=R_{\gamma} L_{\alpha} R_{\alpha \gamma}^{-1}, \Rightarrow L_{\alpha}^{-1} R_{\gamma}^{-1} R_{\beta} L_{\alpha}=R_{\alpha \gamma}^{-1} R_{\alpha \beta}$. Finally, $R_{\beta} J_{\lambda}$ $L_{\beta} L_{\alpha}^{-1} R_{\gamma}^{-1} R_{\beta} L_{\alpha}=J_{\lambda} R_{\alpha \gamma}^{-1} R_{\alpha \beta}^{\gamma}$.

We have the following immediate corollary of the above theorem.

Corollary 1. Let $L$ be a commutative Wilson loop with $\alpha^{2}=e$. Then, for all $\alpha, \beta, \gamma \in L$, we have

(1) $R_{(\alpha, \beta)}=R_{(\alpha)}^{-1}$

(2) $R_{(\alpha, \beta)}^{-1}=\left(R_{\gamma}^{-1}\right)^{3} R_{\beta}^{-1} R_{\beta \gamma}$

(3) $R_{(\alpha, \beta)}=R_{\alpha \beta}^{-1} R_{\beta}^{-1} R_{\alpha}$

The Wilson loop is not ready to accept squaring property unless we select only those which are flexible. The next lemma indicates this notion.

Lemma 3. Let $L$ be the flexible Wilson loop. Then, $(\alpha \beta)^{2}=\alpha^{2} \beta^{2}$ for all $\alpha, \beta \in L$. Moreover, $(\alpha \aleph \beta \aleph)^{2}=\alpha^{2} \aleph \beta^{2} \aleph$ and show that $\left(R_{(\alpha \aleph)^{-1}} L_{\alpha \aleph}, L_{\alpha \aleph}, L_{\alpha \aleph}\right)$ is an autotopism on $L / \aleph$.

Proof. In [2], the Wilson loop $L$ is an extra loop that satisfies the square property $(\alpha \beta)^{2}=\alpha^{2} \beta^{2}$. So, $(\alpha \aleph \beta \aleph)^{2}=(\alpha$ $\beta \aleph)^{2}=(\alpha \beta)^{2} \aleph=\alpha^{2} \aleph \beta^{2} \aleph$. L/ entertains the autotopism $\left(R_{(\alpha \aleph)^{-1}} L_{\alpha \aleph}, L_{\alpha \aleph}, L_{\alpha \aleph}\right)$.

Lemma 4. If $L / \aleph$ is a Wilson loop, then $L$ need not to be a Wilson loop.
Proof. Since $L / \aleph$ is a Wilson loop, so for all $\alpha \aleph, \beta \aleph$, $\gamma \aleph \varepsilon L / \aleph$, we have $\alpha \aleph(\alpha \aleph \beta \aleph)^{\rho}=(\alpha \aleph \gamma \aleph)(\alpha \aleph(\beta \aleph \gamma \aleph))^{\rho} \Rightarrow$ $\alpha \aleph\left((\alpha \beta)^{\rho} \aleph^{\rho}\right)=(\alpha \gamma \aleph) \quad(\alpha(\beta \gamma))^{\rho} \aleph^{\rho} \Rightarrow\left(\alpha(\alpha \beta)^{\rho}\right) \aleph=(\alpha \gamma)(\alpha$ $(\beta \gamma))^{\rho} \aleph$. It implies that $\left(\alpha(\alpha \beta)^{\rho}\right) \neq(\alpha \gamma)(\alpha(\beta \gamma))^{\rho}$, i.e., $L$ is not a Wilson loop.

Theorem 2. The homomorphic image of a Wilson loop is a Wilson loop.

Theorem 3. The direct product of Wilson loops is again a Wilson loop.

Wilson loop can be defined by another way of opposite loop in case of nonassociative finite invertible loop (NAFIL). Thus, we have the following consequence of the above discussion.

Theorem 4. Let $L$ be any NAFIL. A loop $(L, \cdot)$ is a Wilson loop if and only if $(L, \diamond)$ is a Wilson loop, where $(L, \diamond)$ is opposite loop of $(L, \cdot)$. Thus, we have $\alpha \cdot \beta=\beta \diamond \alpha$ for all $\alpha, \beta \in L$.

Proof. Let $(L, \cdot)$ be a Wilson loop, so $\alpha(\alpha \beta)^{\rho}=(\alpha \gamma)(\alpha \cdot \beta \gamma)^{\rho}$, for all $\alpha, \beta$, and $\gamma \in L$. Consider

$$
\begin{aligned}
((\gamma \diamond \beta) \diamond \alpha)^{\lambda} \diamond(\gamma \diamond \alpha) & =\alpha(\alpha \beta)^{\rho} \\
& =\alpha(\alpha \beta)^{\lambda} \\
& =(\beta \diamond \alpha)^{\lambda} \diamond \alpha .
\end{aligned}
$$

It shows that $(L, \diamond)$ is Wilson loop.

Conversely, now we suppose that $(L, \diamond)$ is a Wilson loop,

$$
\begin{aligned}
(\gamma \beta \cdot \alpha)^{\lambda}(\gamma \alpha) & =(\alpha \diamond \gamma) \diamond(\alpha \diamond(\beta \diamond \gamma))^{\lambda} \\
& =(\alpha \diamond \gamma) \diamond(\alpha \diamond(\beta \diamond \gamma))^{\rho} \\
& =\alpha \diamond(\alpha \diamond \beta)^{\rho} \\
& =\alpha \diamond(\alpha \diamond \beta)^{\lambda} \\
& =(\alpha \diamond \beta)^{\lambda} \alpha \\
& =(\beta \alpha)^{\lambda} \alpha .
\end{aligned}
$$

It completes the proof.

We finish this section with the following inclusions. 
Lemma 5. Let $L$ be a Wilson loop. Then, the following statements hold:

(1) $\{$ Wilson loop $\} \subset\{$ Osborn loop $\}$

(2) $\{$ flexible Wilson loop $\} \subset\{$ S-loop $\}$

(3) $\{$ Wilson loop $\} \subset\{$ automorphic inverse property loop $(A I P L)\}$

(4) $\{$ extra loop $\} \subset\{$ Wilson loop $\}$

Proof. First two results are obvious from [2, 6]. For (3), the Wilson loop $\Rightarrow$ the Osborn loop $\Rightarrow$ the Moufang loop$\Rightarrow$ inverse property loop (IPL) $\Rightarrow$ cross inverse property loop $(\mathrm{CIPL}) \Rightarrow$ AIPL.

\section{Holomorphism of Wilson Loop}

In this section, we describe the conditions under which the holomorph of Wilson loop is Wilson loop. Here is the first result.

Lemma 6. If holomorph $H(L)$ of a loop $L$ is Wilson loop, then $L$ is a Wilson loop.

Proof. Since $(I, L)$ is a normal subloop of $H(L)$, moreover $(I, L)$ is isomorphic to $L$, so $L$ is a normal subloop of $H(L)$. Since $H(L)$ is a Wilson loop, so does $L$.

Theorem 5. If $L$ is a Wilson loop, then for all $(\Phi, \alpha)$, $(\xi, \gamma) \in H(L), \alpha, \beta, \gamma \in L$, and $\Phi, \xi \in A U M(L)$, we have $((\Phi, \alpha)(\xi, \gamma))((\Phi, \alpha) \cdot(I, \beta)(\xi, \gamma))^{\rho}=(\Phi, \alpha)((\Phi, \alpha)(I, \beta))^{\rho}$.

Proof. Necessary computations are given below

$$
\begin{aligned}
((\Phi, \alpha)(\xi, \gamma))((\Phi, \alpha) \cdot(I, \beta)(\xi, \gamma))^{\rho} & =((\Phi, \alpha)(\xi, \gamma))\left((\Phi \xi)^{-1},(\alpha \xi \cdot(\beta \xi \cdot \gamma))^{\rho}(\Phi \xi)^{-1}\right) \\
& =\left(I,(\alpha \xi \cdot \gamma) \xi^{-1} \Phi^{-1} \cdot\left(\alpha \Phi^{-1} \cdot\left(\beta \Phi^{-1} \cdot \gamma \xi^{-1} \Phi^{-1}\right)\right)^{\rho}\right) \\
& =\left(I,(\alpha \xi \cdot \gamma) \xi^{-1} \Phi^{-1} \cdot\left(\alpha \Phi^{-1} \cdot\left(\beta \Phi^{-1} \cdot \gamma \xi^{-1} \Phi^{-1}\right)\right)^{\rho}\right) \\
& =\left(I, \alpha \Phi^{-1}\left(\alpha \Phi^{-1} \cdot \beta \Phi^{-1}\right)^{\rho}\right) \\
& =\left(\Phi \Phi^{-1}, \alpha \Phi^{-1} \cdot\left((\alpha \beta) \Phi^{-1}\right)^{\rho}\right) \\
& =(\Phi, \alpha)\left(\Phi^{-1},\left((\alpha \beta)^{\rho} \Phi^{-1}\right)\right) \\
& =(\Phi, \alpha)(\Phi, \alpha \beta)^{\rho} \\
& =(\Phi, \alpha)(\Phi I, \alpha I \cdot \beta)^{\rho} \\
& =(\Phi, \alpha)((\Phi, \alpha)(I, \beta))^{\rho} .
\end{aligned}
$$

Thus, we have the following immediate corollary.

Corollary 1. The holomorph $H(L)$ of a loop $L$ is a Wilson loop if and only if $L$ is a Wilson loop and $A U M(L)$ is trivial group.

Lemma 7. If $H(L)$ is a Wilson loop of a commutative loop $L$, then $J_{\lambda}=J_{\rho}=I$.

Proof. With the aid of the last two results, loop $L$ is commutative AIPL. Therefore, $J_{\lambda}=J_{\rho}=I$.

Lemma 8. Let $U=(L, \cdot)$ and $V=(L, \diamond)$ be two loops and $L$ is a nonempty set. If $H(U)$ and $H(V)$ are isomorphic Wilson loops, then binary operations are coincident.

Proof. Isomorphism of $H(U)$ and $H(V)$ gives $\alpha \delta \diamond \beta \xi=(\alpha \pi \cdot \beta) \delta$ for all $\xi, \delta \in \operatorname{AUM}(V), \alpha, \beta \in L$, and $\pi \in \operatorname{AUM}(U)$. Put $\pi=\xi=\delta=I$, then we have $\alpha I \diamond \beta I=(\alpha$ $I \cdot \beta) I \Rightarrow \alpha \diamond \beta=\alpha \cdot \beta$ for all $\alpha, \beta \in L$. Hence, we have two identical loops $U$ and $V$.

Similarly, we state the following obvious result.

Lemma 9. Let $L$ be any loop with holomorph $H(L) . H(L)$ is Wilson CIPL if and only if $L$ is Wilson CIPL and $\operatorname{AUM}(L)=\{I\}$.

Theorem 6. Let $L$ be a loop with holomorph $H(L) . H(L)$ is Wilson loop if and only if for all $\Psi, \xi \in A U M(L)$, we have

$$
\alpha \xi \cdot((\alpha \Psi \cdot \beta) \xi)^{\rho}=(\alpha \xi \cdot \gamma)((\alpha \Psi \xi)(\beta \xi \cdot \gamma))^{\rho} .
$$

Proof. For all $(\Phi, \alpha),(\Psi, \beta) \in H(L)$, 


$$
\begin{aligned}
(\Phi, \alpha)((\Phi, \alpha)(\Psi, \beta))^{\rho} & =(\Phi, \alpha)\left((\Phi \Psi)^{-1},(\alpha \Psi \cdot \beta)^{\rho}(\Phi \Psi)^{-1}\right) \\
& =\left(\Phi \cdot \Psi^{-1} \Phi^{-1}\right), \alpha \Psi^{-1} \Phi^{-1} \cdot(\alpha \Psi \cdot \beta)^{\rho}\left(\Psi^{-1} \Phi^{-1}\right) \\
& =\left(\Phi \cdot \Psi^{-1} \Phi^{-1},\left(\alpha(\alpha \Psi \cdot \beta)^{\rho}\right) \Psi^{-1} \Phi^{-1}\right), \\
((\Phi, \alpha)(\xi, \gamma))((\Phi, \alpha) \cdot(\Psi, \beta)(\xi, \gamma))^{\rho} & =(\Phi \xi, \alpha \xi \cdot \gamma)((\Phi, \alpha) \cdot(\Psi \xi, \beta \xi \cdot \gamma))^{\rho} \\
& =(\Phi \xi, \alpha \xi \cdot \gamma)\left((\Phi \cdot \Psi \xi)^{-1},(\alpha \Psi \xi \cdot(\beta \xi \cdot \gamma))^{\rho}(\Phi \cdot \Psi \xi)^{-1}\right) \\
& =\left((\Phi \xi)\left(\xi^{-1} \Psi^{-1} \cdot \Phi^{-1}\right),(\alpha \xi \cdot \gamma)\left(\xi^{-1} \Psi^{-1} \cdot \Phi^{-1}\right)\right. \\
& =(\alpha \Psi \xi \cdot(\beta \xi \cdot \gamma))^{\rho}\left(\xi^{-1} \Psi^{-1} \cdot \Phi^{-1}\right) \\
& =\left(\Phi \cdot \Psi^{-1} \Phi^{-1},\left((\alpha \xi \cdot \gamma)(\alpha \Psi \xi \cdot(\beta \xi \cdot \gamma))^{\rho}\right)\left(\xi^{-1} \Psi^{-1} \cdot \Phi^{-1}\right)\right) .
\end{aligned}
$$

Given hypothesis is

$$
\begin{aligned}
\alpha \xi \cdot((\alpha \Psi \cdot \beta) \xi)^{\rho} & =(\alpha \xi \cdot \gamma)((\alpha \Psi \xi)(\beta \xi \cdot \gamma))^{\rho} \\
\alpha(\alpha \Psi \cdot \beta)^{\rho}(\Phi \Psi)^{-1} & =(\alpha \xi \cdot \gamma)(\alpha \Psi \xi \cdot(\beta \xi \cdot \gamma))^{\rho} \xi^{-1}(\Phi \Psi)^{-1} \\
\alpha(\alpha \Psi \cdot \beta)^{\rho}\left(\Psi^{-1} \Phi^{-1}\right) & =(\alpha \xi \cdot \gamma)(\alpha \Psi \xi \cdot(\beta \xi \cdot \gamma))^{\rho}\left(\xi^{-1}\left(\Psi^{-1}\right) \cdot \Phi^{-1}\right) .
\end{aligned}
$$

Thus, equation (9) becomes

$$
\begin{aligned}
& ((\Phi, \alpha)(\xi, \gamma))((\Phi, \alpha) \cdot(\Psi, \beta)(\xi, \gamma))^{\rho} \\
& \quad=\left(\Phi \cdot \Psi^{-1} \Phi^{-1},\left(\alpha(\alpha \Psi \cdot \beta)^{\rho}\right) \Psi^{-1} \cdot \Phi^{-1}\right) .
\end{aligned}
$$

We summarize the above discussion in the following manner.

Theorem 7. Let L be a loop with holomorph $H(L)$. Then, the following are equivalent:

(1) $H(L)$ is Wilson loop

(2) $L$ is Wilson loop with trivial group $\operatorname{AUM}(L)$

(3) $\left.\alpha \xi \cdot((\alpha \Psi \cdot \beta) \xi)^{\rho}\right)=(\alpha \xi \cdot \gamma)((\alpha \Psi \xi)(\beta \xi \cdot \gamma))^{\rho}$ for all $\Psi, \xi \in A U M(L)$

Lemma 10. Let $H(L)$ be the holomorph of the loop L. Then, $\operatorname{AUM}(L)$ has right action on $H(L)$ but not left.

Proof. We define $(\Phi, \alpha)^{\delta}=(\Phi \delta, \alpha \delta)$ for all $(\Phi, \alpha) \epsilon$ $H(L), \delta \in \operatorname{AUM}(L)$.

$$
\begin{aligned}
(\Phi, \alpha)^{g} & =(\Phi g, \alpha g), \\
\left((\Phi, \alpha)^{g}\right)^{h} & =((\Phi g) h,(\alpha g) h) \\
& =(\Phi(g h), \alpha(g h)) \\
& =(\Phi, \alpha)^{g h} .
\end{aligned}
$$

For all $g, h \in \operatorname{AUM}(L)$. Moreover,

$$
(\Phi, \alpha)^{I}=(\Phi I, \alpha I)=(\Phi, \alpha)
$$

So, $\operatorname{AUM}(L)$ has right action on $H(L)$. Clearly, $(\Phi, \alpha)^{g h} \neq(\Phi, \alpha)^{h g}$. $\operatorname{AUM}(L)$ has not left action on $H(L)$.
Remark 1. AUM $(L)$ has both the left and right actions on Wilson loop $H(L)$.

Proposition 3. If $H(L)$ has the Wilson loop, then $J_{\lambda}$, $J_{\rho} \in S_{1}(L)$, where $S_{1}(L)$ is the group of weak inverse permutations of $L$.

Proof. Since $H(L)$ is Wilson loop, so $\operatorname{AUM}(L)$ is trivial. By Wilson identity, $\alpha(\alpha \beta)^{\rho}=(\alpha \gamma)(\alpha \cdot \beta \gamma)^{\rho}$ for all $\alpha, \beta$, and $\gamma \in L$. Put $\alpha=e$, then $\beta^{\rho}=\gamma(\beta \gamma)^{\rho}, L$ is WIPL. $J_{\rho}^{2}$ is an automorphism of this loop by [5], therefore $J_{\rho}^{2}$ is an identity mapping because $\operatorname{AUM}(L)$ is trivial. To show $J_{\rho} \in S_{1}(L)$, we verify $\alpha^{\rho}=\left(\left(\alpha J_{\rho}\right)^{\rho}\right) J_{\rho}$.

$$
\begin{aligned}
\left(\left(\alpha J_{\rho}\right)^{\rho}\right) J_{\rho} & =\left(\alpha J_{\rho}\right) J_{\rho}^{2} \\
& =(\alpha) J_{\rho} \\
& =\alpha^{\rho} .
\end{aligned}
$$

It implies that $J_{\rho}$ is the weak right inverse permutation, so $J_{\rho} \in S_{\rho}(L)$. It can be shown easily

$$
S_{\rho}(L)=S_{\lambda}(L)=S_{1}(L) .
$$

Therefore, $J_{\rho} \in S_{1}(L)$. Similarly, we can prove $J_{\lambda} \in S_{1}(L)$.

Remark 2. Let $L$ be the NAFIL with trivial group $\operatorname{AUM}(L, \diamond)=\operatorname{AUM}(L, \cdot) \cdot H(L, \cdot)$ is Wilson loop if and only if $H(L, \diamond)$ is Wilson loop.

\section{Data Availability}

The data used to support the findings of this study are included within the article.

\section{Conflicts of Interest}

The authors declare that there are no conflicts of interest regarding the publication of this paper.

\section{Authors' Contributions}

Han Li wrote the final version of the paper and arranged funding for this paper, M. Nadeem proved the main results, 
M. A. Ali wrote the first version of the paper, and H. Mutee ur Rehman proposed the problem and supervised this work.

\section{References}

[1] E. L. Wilson, "A class of loops with the isotopy-isomorphy property," Canadian Journal of Mathematics, vol. 18, pp. 589-592, 1966.

[2] E. G. Goodaire and D. A. Robinson, "Some special conjugacy closed loops," Canadian Mathematical Bulletin, vol. 33, no. 1, pp. 73-78, 1990.

[3] R. H. Bruck, "Contributions to the theory of loops," Transactions of the American Mathematical Society, vol. 55, pp. 245-354, 1944.

[4] T. G. Jaiyeola, "An holomorphic study of the Smarandache concept in loops," Scientia Magna Journal, vol. 2, no. 1, pp. 1-8, 2006.

[5] J. Osborn, "Loops with the weak inverse property," Pacific Journal of Mathematics, vol. 10, no. 1, pp. 295-304, 1960.

[6] A. D. Keedwell, "Crossed-inverse Quasigroups with long inverse cycles and applications to cryptography," Australasian Journal of Combinatorics, vol. 20, pp. 241-250, 1999. 